\title{
Indexing of Satellite Images With Different Resolutions by Wavelet Features
}

\author{
Bin Luo, Jean-François Aujol, Yann Gousseau, and Saïd Ladjal
}

\begin{abstract}
Space agencies are rapidly building up massive image databases. A particularity of these databases is that they are made of images with different, but known, resolutions. In this paper, we introduce a new scheme allowing us to compare and index images with different resolutions. This scheme relies on a simplified acquisition model of satellite images and uses continuous wavelet decompositions. We establish a correspondence between scales which permits us to compare wavelet decompositions of images having different resolutions. We validate the approach through several matching and classification experiments, and we show that taking the acquisition process into account yields better results than just using scaling properties of wavelet features.
\end{abstract}

Index Terms - Image indexing, resolution invariance, satellite images, wavelet features.

\section{INTRODUCTION}

$\mathbf{O}$ VER the last years, space agencies have built up massive image databases. For example, the CNES (the French space agency) gets each day several terabytes of data from its satellites. These institutions need efficient tools to index and search their image databases. One particularity of satellite image databases, compared to, e.g., natural image databases, is that they are constituted by images with different but known resolutions ${ }^{1}$ depending on the satellite which acquires them. In contrast, the relationship between the size of objects and pixels is usually unknown for natural images. Moreover, this relationship depends on the position of objects in the scene, so that the notion of resolution itself has little general meaning for natural images. This obvious fact made it necessary to develop scale invariant local features for many computer vision tasks, see, e.g., [1]. For the indexing of texture, it makes sense to assume a uniform resolution through the image. Since this resolution is usually unknown, many scale invariant indexing schemes have been developed, see, e.g., [2]-[4], and [5] for a review. Our purpose in this paper is quite different. First, the resolution of satellite images is usually a known parameter, at least if we neglect tilts of the optical device and if we assume that the scene

Manuscript received October 19, 2007; revised March 11, 2008. First published June 24, 2008; last published July 11, 2008 (projected). The associate editor coordinating the review of this manuscript and approving it for publication was Dr. Pier Luigi Dragotti.

B. Luo is with GIPSA-Lab, Grenoble, France.

Y. Gousseau and S. Ladjal are with TELECOM ParisTech, LTCI CNRS, Paris, France.

J.-F. Aujol is with CMLA, ENS Cachan, CNRS, UniverSud, Cachan, France. Color versions of one or more of the figures in this paper are available online at http://ieeexplore.ieee.org.

Digital Object Identifier 10.1109/TIP.2008.925367

${ }^{1}$ By resolution, we mean the true pixel size in meter. being captured is approximately flat. Therefore, our goal is to be able to compare two images knowing their resolution difference. Second, a resolution change is more complicated than just a scale change, since it usually involves an optical device and an imaging captor. In a previous work, [6], [7], this process was modeled as a convolution followed by a sampling and its effect on the computation of a characteristic scale was studied. In this paper, we make use of the same model and propose a scheme to compare features extracted from images at different resolutions. Observe that several works have been performed to extract image features that are invariant with respect to resolution changes [8], [9]. Again, our purpose is quite different since we wish to be able to compare images with different but known resolution. Many features have been proposed to index satellite images [10]-[14]. In this work, we only consider mono-spectral images and classically choose to index them using texture features. In particular, wavelet features have been proved suitable for texture indexing or classification [15]-[22]. Wavelet features have already been used for indexing remote-sensing images in [23]. The aim of the proposed approach is to investigate the interplay between resolution and wavelet features and to propose a scheme for the comparison of images with different resolutions. Preliminary results of the present work were presented in [24].

The plan of the paper is the following. In Section II, a simplified model for the acquisition of satellite images is introduced. In Section III, we recall how the marginals of wavelet coefficients can be used for the indexing of images. In Section IV, a method is given to compare features extracted at different resolutions. In Section V, the dependence of features upon resolution is checked using satellite images from the CNES and the proposed scheme is validated through classification experiments. We then conclude in Section VI.

\section{Model of THE AcQuisition Process}

A digital image $f_{r}$ at resolution $r$ is obtained from a continuous function $f$ (representing the scene under study) through an optical device and a digital captor. Neglecting contrast changes and quantization, the effect of the imaging device can be modeled as follows:

$$
f_{r}=\Pi_{S_{r}}(G * f)+n
$$

where $G$ is the convolution kernel, $S_{r} \subset \mathbb{Z}^{2}$ the sampling grid at resolution $r, \Pi_{S_{r}}$ the Dirac comb on $S_{r}$ and $n$ the noise. In what follows, we will take interest in the effect of the acquisition model on the wavelet coefficients of $f_{r}$. Therefore, assuming that we will neglect coefficients at the smallest scales, we will assume that $n=0$. Moreover, we will assume that 
$S_{r}=r \mathbb{Z}^{2}$, that is a regular and square sampling grid with step $r$. We, thus, neglect the satellite vibrations and scan acquisition. Last and more importantly, according to [25], the response of an ideal optic aperture can be accurately approximated by a Gaussian kernel. We will, therefore, assume that $G$ is an isotropic Gaussian kernel, thus neglecting the specificity of the satellite optics, the real response of the captor and motion blur. This is probably the strongest assumption made in this paper. The motivation behind it is mainly the tractability of forecoming computations, as will become clear soon. Last, we assume that the standard deviation of the kernel is proportional to the resolution. In the experimental section, we will check that these assumptions are not too restrictive by considering real satellite images. To summarize, we assume the following acquisition model:

$$
f_{r}=\Pi_{r}\left(f * k_{r p}\right)
$$

where

$$
k_{r p}(x, y)=\frac{1}{2 \pi r^{2} p^{2}} \exp \left(-\frac{x^{2}+y^{2}}{2 r^{2} p^{2}}\right)
$$

$\Pi_{r}$ is the Dirac comb on $r \mathbb{Z}^{2}$, that is

$$
\Pi_{r}=\sum_{i, j \in \mathbb{Z}} \delta_{(i r, j r)}
$$

and the parameter $p$ is a characteristic of the acquisition process, which characterizes the width of the convolution kernel: the larger the value of $p$, the more blurred the image.

\section{WAVELET FEATURES FOR TEXTURE INDEXATION}

Based on empirical observations, S. Mallat [27] proposed to model the marginals of wavelet coefficients of natural images by generalized Gaussian distributions (GGD). That is, writing $h(w)$ for the density of the distribution of coefficients $w$ at some scale and orientation

$$
h(w)=K e^{-(|w| / \alpha)^{\beta}} .
$$

It is shown in [15]-[17] that the parameters $\alpha$ and $\beta$ of GGD can be used as efficient features for texture indexing and classification. It is possible to compute these parameters from the estimation of the first and second order moments of $|w|$ [27]: we denote them, respectively, by $m_{1}=\int|w| h(w) d w$ and $m_{2}=$ $\int w^{2} h(w) d w$. More precisely, $m_{1}=\alpha \Gamma(2 / \beta) \Gamma(1 / \beta)^{-1}$ and $m_{2}=\alpha^{2} \Gamma(3 / \beta) \Gamma(1 / \beta)^{-1}$, where $\Gamma$ stands for the Gamma function.

In this paper, for simplicity, we address the problem of relating features $m_{1}$ and $m_{2}$ to resolution changes. Adapting the results to $\alpha$ and $\beta$ is then straightforward and this can be useful when using the Kullback-Leibler distance in a classification task, see [16].

In order not to be restricted to dyadic resolution changes, continuous wavelet transform [28] is used instead of the more classical discrete wavelet transform. Moreover, we consider mother wavelets obtained as derivatives of a Gaussian kernel in horizontal, vertical, and diagonal directions. This important assump-

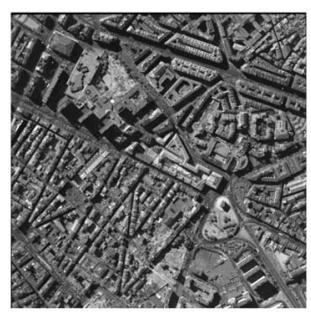

(a)

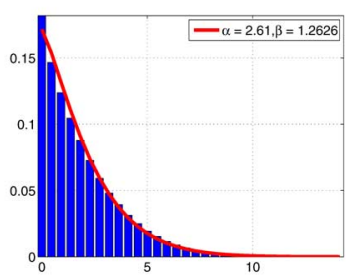

(b)
Fig. 1. (a) Image of Marseille at resolution 0.707 m (ㄷ CNES). (b) Histogram (blue bars) of wavelet coefficients of image (a) at scale 5 (horizontal) and the approximation by GGD (red curve).

tion is motivated by the simplified model for resolution changes presented in the previous section, as will be shown by the computations of Section IV-A.

Fig. 1 shows a histogram of absolute values of wavelet coefficients, illustrating the soundness of the use of GGDs to model such distributions.

\section{WAVElet FeAtures AND Resolution ChangeS}

\section{A. Resolution Invariance}

a) Notations: The discrete version of the Gaussian kernel with standard deviation $t$ ( $t$ being given in pixels) is denoted by $\tilde{k}_{t}$. Let us define the discrete wavelet coefficients as (recall that the wavelets we use are derivatives of the Gaussian kernel)

$$
w_{q, r, t}=\Delta_{q} \tilde{k}_{t} \tilde{*} f_{r}=\tilde{k}_{t} \tilde{*} \Delta_{q} f_{r}
$$

where $q \in\{0,1,2,3\}, \Delta_{q}$ stands for the difference between adjacent pixels in the horizontal $(q=0)$, vertical $(q=1)$ or diagonal $(q=2,3)$ direction, $\tilde{*}$ stands for the discrete convolution operation.

b) Resolution Invariance: Recall that the image $f_{r}$ at resolution $r$ is obtained as $f_{r}=\Pi_{r}\left(k_{r p} * f\right)$. From (4), we, therefore, have

$$
\begin{aligned}
w_{q, r, t} & =\tilde{k}_{t} \tilde{*}_{q} \Pi_{r}\left(k_{r p} * f\right) \\
& \approx \tilde{k}_{t} \tilde{*} \Pi_{r}\left(r \partial_{q}\left(k_{r p} * f\right)\right)
\end{aligned}
$$

where $\partial_{q}$ is the continuous directional derivative at orientation $q$. This last approximation is detailed in the Appendix.

Next, we assume that the inversion between the convolution and the sub-sampling is appropriate for nonaliased images such as $k_{r p} * f$ when $p$ is at least, say, $1 / 2$. The validity of this assumption on real images has been checked in [7]. Assuming that $\tilde{k}_{t} \approx k_{r t}$ (see [7]), and that the continuous and discrete convolutions are equivalent, we have

$$
w_{q, r, t} \approx r \Pi_{r}\left(k_{r t} * k_{r p} * \partial_{q} f\right) .
$$

Using the semi-group property of the Gaussian kernel, it can be deduced that

$$
\frac{w_{q, r, t}}{r} \approx \Pi_{r}\left(k_{r \sqrt{t^{2}+p^{2}}} * \partial_{q} f\right) .
$$

The accuracy of this approximation will be computed in the Appendix. 
Assume now that we have two images $f_{r_{1}}$ and $f_{r_{2}}$ of the same scene at resolutions $r_{1}$ and $r_{2}$. From (7), we deduce that if we choose scales $t_{1}$ and $t_{2}$ such that

$$
r_{1} \sqrt{t_{1}^{2}+p^{2}}=r_{2} \sqrt{t_{2}^{2}+p^{2}}
$$

then

$$
\begin{aligned}
& m_{1}\left(q, r_{1}, t_{1}\right) / r_{1} \approx m_{1}\left(q, r_{2}, t_{2}\right) / r_{2} \\
& m_{2}\left(q, r_{1}, t_{1}\right) / r_{1}^{2} \approx m_{2}\left(q, r_{2}, t_{2}\right) / r_{2}^{2}
\end{aligned}
$$

with

$$
\begin{aligned}
& m_{1}(q, r, t)=\frac{1}{n_{r}} \sum\left|w_{q, r, t}\right| \\
& m_{2}(q, r, t)=\frac{1}{n_{r}} \sum\left|w_{q, r, t}\right|^{2}
\end{aligned}
$$

where $n_{r}$ is the size of the discrete image $f_{r}$ and the sum is taken over the image domain (notice that, since we use a continuous wavelet transform, $w$ has the same size as $f_{r}$ ). These equalities (9), (10) permits to compare wavelet features from $f_{1}$ and $f_{2}$. In what follows, we denote by $\Theta_{q, r, t}=\left\{m_{1}(q, r, t), m_{2}(q, r, t)\right\}$ the wavelet features at scale $t$ and direction $q$ extracted from $f_{r}$.

c) Remark About the Naive Choice $p=0.0$ : A naive assumption could be made that for the same scene $f$, if we keep

$$
r \times t=C
$$

where $C$ is a constant, the parameter set is also constant (after the correct normalization). However, this assumption is not sufficient because it approximates the resolution change by a simple zoom, which is not consistent with the acquisition process modeled in Section II. We will see in Section V that such a naive choice leads to poor numerical results compared with the use of (8). In what follows, we will call "naive choice" the use of $p=0$ in (8).

d) Tuning of $p$ : To use (8), one needs to know the value of $p$. This is a characteristic of the acquisition process [see (1)]. This can, therefore, be tabulated once for each satellite. Observe that if one considers two images $f_{r_{1}}$ and $f_{r_{2}}$ with different resolutions, it is very likely that they originate from different satellites and that the corresponding values of $p$ be different. In this case, writing $p_{i}$ for the value of $p$ corresponding to resolution $r_{i}$ $(i=1,2)$ it is straightforward to show that $(8)$ can be generalized to

$$
r_{1} \sqrt{t_{1}^{2}+p_{1}^{2}}=r_{2} \sqrt{t_{2}^{2}+p_{2}^{2}} .
$$

This equality again ensures that approximations (9) and (10) hold and permits the comparison of wavelet features from $f_{r_{1}}$ and $f_{r_{2}}$. In what follows, for the sake of simplicity, we will assume that $p_{1}=p_{2}$ and, therefore, use (8).

\section{B. Wavelet Features and Resolution}

As explained in the introduction, the aim of this paper is to propose a way to compare the features originating from two images with different and known resolutions. One way to achieve this is to modify the features (i.e., the first and second order moments $m_{1}$ and $m_{2}$ ) extracted at resolution $r_{1}$ to compare them with the features extracted at resolution $r_{2}$. Assume that we have $f_{r_{1}}$ the image at resolution $r_{1}$ of a given scene, and that we want to predict its features at resolution $r_{2}$. From (7)-(10), we deduce the following scheme.

- Compute the wavelet coefficients for $f_{r_{1}}$ at scales $t_{i}, i=$ $1,2,3, \ldots, N$.

- Estimate the parameters $\Theta_{q, r_{1}, t_{i}}$ from the wavelet coefficients at scales $t_{i}$ for resolution $r_{1}$.

- For resolution $r_{2}$, compute the scales $t_{i}^{\prime}$ corresponding to $t_{i}$ according to [see (8)]

$$
t_{i}^{\prime}=\sqrt{\frac{r_{1}^{2}}{r_{2}^{2}}\left(t_{i}^{2}+p^{2}\right)-p^{2}} .
$$

- Define $\tilde{\Theta}_{q, r_{2}, t_{i}^{\prime}}=\Theta_{q, r_{1}, t_{i}}$ at scales $t_{i}^{\prime}$.

By using such an algorithm, it is now possible to compare images taken at different resolutions and, for instance, to train classification methods on a set of images at only one resolution and to apply the recognition criteria to images at different resolutions.

\section{EXPERIMENTS AND RESULTS}

\section{A. Image Database}

In the following sections, we experimentally validate the proposed scheme for comparing wavelet features. These experiments are carried on an image database provided by the CNES (the French space agency). This database is made of various scenes (such as fields, forests and cities). Each scene has been acquired as an aerial image at resolution $0.25 \mathrm{~m}$. Then, for each scene, the CNES has simulated images at various resolutions, using realistic satellite modeling. The available resolutions range from 0.5 to $4 \mathrm{~m}$, according to a geometric progression with ratio $2^{1 / 6}$. In Fig. 2, some examples of the images from the database are shown. It is important at this point to note that convolution kernels used by the CNES are not Gaussian. However, we will see that the approximate acquisition model of Section II yields good numerical results. In what follows, we will use the acquisition model (1) with a value $p=1.3$. This value has been chosen as the value yielding the best numerical results (among values ranging from 1 to 2 by steps of 0.1 ) and it also corresponds to a rough approximation of the kernel used by the CNES.

\section{B. Validity of the Prediction Scheme}

First, we check the validity of (8)-(10) by plotting numerical values of features $m_{1}$ and $m_{2}$ as a function of the resolution.

In Fig. 3(d)-(f) [resp. (g)-(i)], graphs of $m_{1}(q, r, t) / r$ [resp. $\left.\left.m_{2}(q, r, t) / r^{2}\right)\right]$ as functions of $r$ are presented when $r t$ is kept constant [that is, when using the naive normalization of (11) and when $r \sqrt{t^{2}+p^{2}}$ with $p=1.30$ is kept constant; see (8)]. The resolution $r$ ranges from 0.50 to $4 \mathrm{~m}$. For the image at resolution $0.50 \mathrm{~m}$ (the highest available resolution), $m_{1}$ and $m_{2}$ are computed at scale 16 in the horizontal direction. It may be seen that using (11) (that is forgetting the convolution step in the model of 


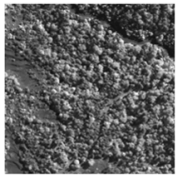

(a)

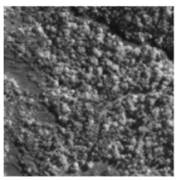

(e)

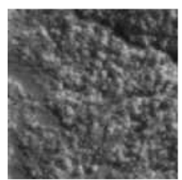

(i)

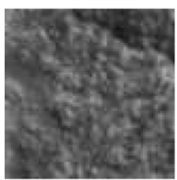

(m)

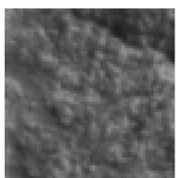

(q)

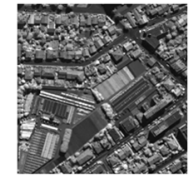

(b)

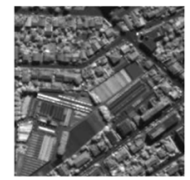

(f)

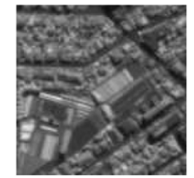

(j)

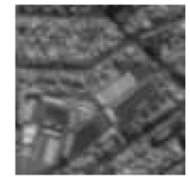

(n)

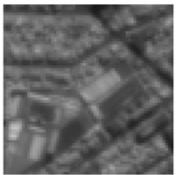

(r)

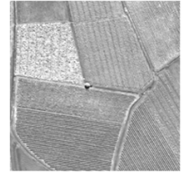

(c)

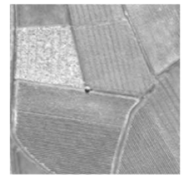

(g)

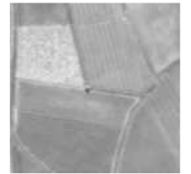

(k)

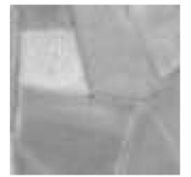

(o)

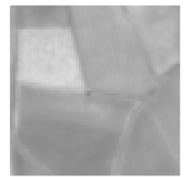

(s)

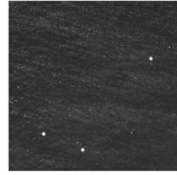

(d)

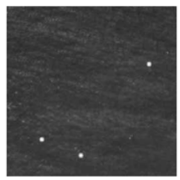

(h)

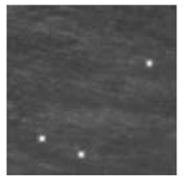

(1)

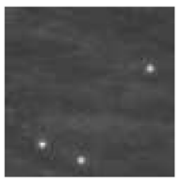

(p)

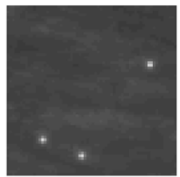

(t)
Fig. 2. Image samples from the database provided by the CNES: (a)-(d) Images at resolution $0.5 \mathrm{~m}$; (e)-(h) images at resolution $1.0 \mathrm{~m}$; (i)-(1) images at resolution $2.0 \mathrm{~m}$; (m)-(p) images at resolution $3.175 \mathrm{~m}$; (q)-(t) images at resolution $4.0 \mathrm{~m}$. From left to right, classes of the images are: field, city, forest, and sea.

resolution change) does not yield a constant parameter set, especially when the resolution change is large. In contrast, using (8) yields fairly constant values.

Next, we compare values of $m_{1}$ and $m_{2}$ computed on an image with resolution $3.175 \mathrm{~m}$ to values of $m_{1}$ and $m_{2}$ computed on a image of the same scene with resolution $1 \mathrm{~m}$ and then predicted for a resolution of $3.175 \mathrm{~m}$. We perform this comparison for various scales. Fig. 4(a)-(c) [resp. (d)-(f)] show the values of $m_{1}$ (respectively, $m_{2}$ ) at various scales (scale is on the horizontal axis) in solid blue line for three different scenes. In the same figure, $m_{1}$ (resp. $m_{2}$ ) predicted from a resolution of $1 \mathrm{~m}$ for a resolution of $3.175 \mathrm{~m}$ according to the scheme presented in Section IV-B are displayed by a solid red line. The two solid lines almost perfectly coincide. In dashed line, are plotted the values of $m_{1}$ (respectively $m_{2}$ ) predicted from a resolution of $1 \mathrm{~m}$ for a resolution of $3.175 \mathrm{~m}$ by the same scheme of Section IV-B, except that (13) is replaced by $t_{i}^{\prime}=r_{1} t_{i} / r_{2}$. This corresponds to what we called the naive choice, neglecting convolutions in the resolution change. It can be seen that in this case, the guessed values of $m_{1}$ and $m_{2}$ are less accurate, especially for small scales. These experiments validate the scheme proposed in Section IV-B and suggests that it is necessary to take into account the convolution step in resolution changes to be able to compare features computed on images with different resolution. The next section investigates how the accuracy of

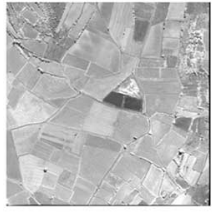

(a)

sout $-m,-$

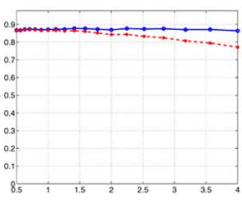

(d)

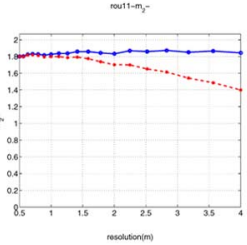

(g)

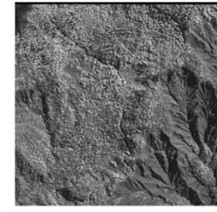

(b)

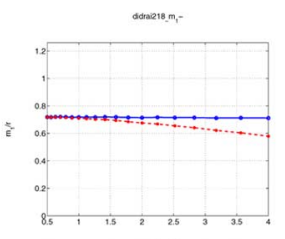

(e)

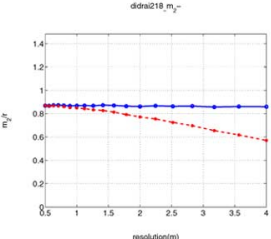

(h)

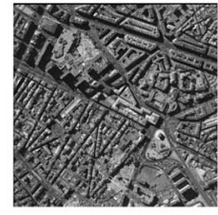

(c)

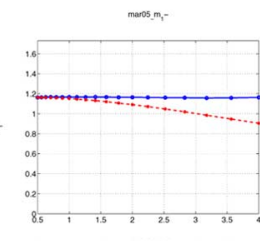

(f)

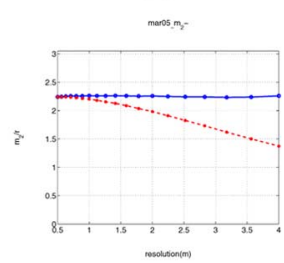

(i)
Fig. 3. (a)-(c) Three images (CCNES); (d)-(f) graphs of $m_{1}(q, r, t) / r$ (with $q=0$ ) as a function of $r$; (g)-(i) graphs of $m_{2}(q, r, t) / r^{2}$ (with $q=0$ ) as a function of $r$. On all these graphs, solid lines correspond to the case where $r \sqrt{t^{2}+p^{2}}$ is kept constant (with $p=1.3$ ), and dashed lines correspond to the case the case where $r t$ is kept constant. One observes that using (8) yields fairly constant values, whereas using (11) does not.

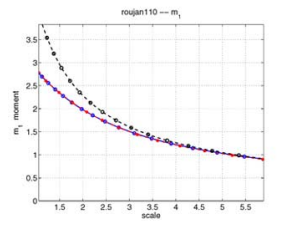

(a)

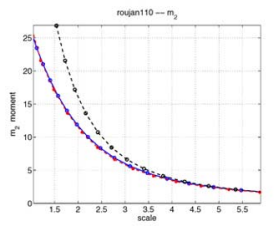

(d)

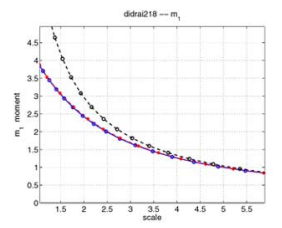

(b)

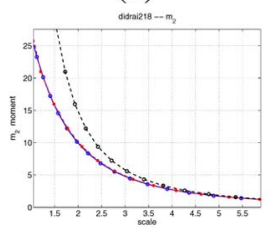

(e)

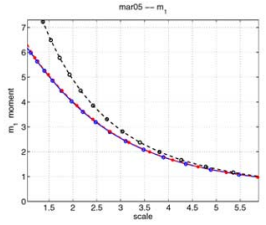

(c)

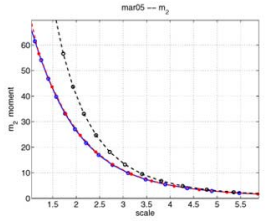

(f)
Fig. 4. Values of $m_{1}$ (a)-(c) and $m_{2}$ (d)-(f) for various scales (scale corresponds to the horizontal axis). In solid red line are displayed the values computed directly on images with resolution $3.175 \mathrm{~m}$ (the ground truth in this experiment). In solid blue line, are displayed the values first computed on the images with resolution $1 \mathrm{~m}$ and then predicted using the scheme presented in Section IV-B. Both solid lines almost perfectly coincide, showing the accuracy of the scheme. Dash lines show the results obtained by using the scheme proposed in Section IV-B but replacing (13) with $t_{i}^{\prime}=r_{1} t_{i} / r_{2}$ (the naive normalization). Observed and predicted values do not correspond anymore in this case.

the proposed scheme permits the classification of images at different resolutions.

\section{Classification}

a) Classified Database: We have manually built a classified database based on the sequences of images provided by the CNES. This database contains 366 scenes observed on urban areas (Marseille and Toulouse), rural areas (Roujan), forests (Didrai) and the sea. For each scene, there are five different images corresponding to five different resolutions $(0.5,1,2,3.175$, 
TABLE I

Classification Results ObTained With Wavelet Features EXTRacted IN Four DiRECTIONS AT 21 SCALES $\left(t=\left\{2^{i / 6} \mid i=0,1, \ldots, 20\right\}\right)$. THE LEARNING SET IS MADE OF IMAGES AT ReSOlUtion $4 \mathrm{~m}$

\begin{tabular}{|c|c|c|}
\hline Resolution & error $(p=1.3)$ & error $(p=0)$ \\
\hline $0.5 \mathrm{~m}$ & $0.00 \%$ & $21.86 \%$ \\
\hline $1 \mathrm{~m}$ & $0.00 \%$ & $19.95 \%$ \\
\hline $2 \mathrm{~m}$ & $0.00 \%$ & $10.66 \%$ \\
\hline $3.175 \mathrm{~m}$ & $0.00 \%$ & $1.91 \%$ \\
\hline
\end{tabular}

and $4 \mathrm{~m})$. The scenes are manually divided into four sets: city (199 scenes), fields (134 scenes), forests (23 scenes), and sea (ten scenes).

b) Experiment: The first experiment carried out on this database is classification. Images at the resolution of $4 \mathrm{~m}$ are used as a learning set for training the classifier. The aim is to find the classes of the images at resolutions other than $4 \mathrm{~m}$. Wavelet features $\left(m_{1}\right.$ and $\left.m_{2}\right)$ are at first extracted from all the images by applying Gaussian derivatives at different scales in four directions (horizontal, vertical and diagonal). The features extracted from the images at a resolution of $4 \mathrm{~m}$ over 21 scales $\left(t=\left\{2^{i / 6}, i=0,1, \ldots, 20\right\}\right)$ are used to train the classifier. Therefore, the dimension of the feature space is $2 \times 4 \times 21=$ 168. The features extracted from the other resolutions are predicted at a resolution of $4 \mathrm{~m}$ by using the scheme presented in Section IV-B. For comparison, we set the value $p$ respectively equal to 1.3 (using the acquisition model) and 0.0 (the naive approach). We then classify the images at resolutions other than 4 $\mathrm{m}$ with the predicted features.

The classifier we used is simply the nearest neighbor classification algorithm. For a given image A, the classifier search for its nearest neighbor $\mathrm{B}$ in the training set and affect to $\mathrm{A}$ the class of B. As a distance between features, we use the Euclidean distance, after normalizing each coordinate by its variance.

c) Results: The classification results are shown in Table I. It can be observed that with the naive approach, the classification errors increase rapidly when the resolution gets away from $4 \mathrm{~m}$. This shows numerically that the naive approach $(p=0)$ is not a good choice for classification purpose. On the contrary, when the acquisition model is taken into consideration (i.e., $p=1.3$ ), there is no error. This is due to the fact that the prediction scheme is very accurate, as we have shown in Section V-B. Errors are small enough not to switch from one class to another when changing the resolution.

d) Influence of the Number of Features: The classification results presented in Table I are obtained from features in a space with relatively large dimension (168 values for each image). We, therefore, study the effect of a dimension reduction. In Table II, are shown the classification results obtained when performing the same experiment as in Table I, using the wavelet features $\left(m_{1}\right.$ and $\left.m_{2}\right)$ at only three scales $(t=1,2,4)$. In this case, the dimension of the feature space is $2 \times 4 \times 3=24$ and it can be observed that the errors remain similar.

e) Comparison With Other Features: In order to further investigate the effect of resolution changes on classification tasks, we perform an experiment using Haralick features. Haralick [29] has proposed features based on the statistics of co-occurrence matrices of images. These features are proved to be very efficient for indexing textures. Co-occurrence matrices are defined as the empirical joint distribution of the gray values of
TABLE II

Classification Results ObTainEd With WaVELET Features EXTRACTED In Four Directions at Three Scales $(t=1,2,4)$. The LEARnING SET IS MAdE OF IMAGES AT RESOLUTION $4 \mathrm{M}$

\begin{tabular}{|c|c|c|}
\hline Resolution & error $(p=1.3)$ & error $(p=0)$ \\
\hline $0.5 \mathrm{~m}$ & $0.55 \%$ & $26.50 \%$ \\
\hline $1 \mathrm{~m}$ & $0.00 \%$ & $24.59 \%$ \\
\hline $2 \mathrm{~m}$ & $0.00 \%$ & $12.84 \%$ \\
\hline $3.175 \mathrm{~m}$ & $0.27 \%$ & $4.92 \%$ \\
\hline
\end{tabular}

TABLE III

Classification Results Obtained With Haralick Features. The Distance PARAMETER For CAlCUlating CO-OCCURRENCE MATRICES VARIES ACCORDing to the Resolution of IMAge. The Learning Set IS MADE OF IMAGES AT RESOLUTION 4 M

\begin{tabular}{|c|c|c|}
\hline Resolution & distance & error \\
\hline $0.5 \mathrm{~m}$ & 24 & $31.9 \%$ \\
\hline $1 \mathrm{~m}$ & 12 & $22.4 \%$ \\
\hline $2 \mathrm{~m}$ & 6 & $13.6 \%$ \\
\hline $3.175 \mathrm{~m}$ & 4 & $10.1 \%$ \\
\hline
\end{tabular}

pixels in some direction $\theta$ and at some distance $d$. The considered directions are horizontal, vertical and two diagonal directions. The distance between the pixel pairs can be considered as a scale parameter. For images at different resolutions, it is, therefore, natural to compute co-occurrence matrices with different distances. In our experiment, we set $d=3,4,6,12$, 24 , respectively, for images at resolutions $4,3.175,2,1$, and $0.5 \mathrm{~m}$. These values ensure that $d \times r$ is a constant. The Haralick features are composed of 13 statistical values calculated from each matrix and the mean and standard deviation values through the four directions. Therefore, the total feature dimension is $(4+2) \times 13=78$.

Fig. 3 shows the classification results obtained with Haralick features. Our purpose here is not to compare directly these results with the results obtained in the previous section. Indeed, results obtained with wavelet features are better, but we did not take full advantages of co-occurrence matrices since only one scale is used for each image. The interesting point is to notice how fast the classification results decrease with the change of resolution, therefore showing the inability of Haralick features to handle such changes. Observe that the approach taken here is similar to the naive choice of previous sections (approximating a resolution change through a zoom). Due to the nonlinear nature of co-occurrence matrices, the approach proposed in the case of wavelet features is not adaptable to Haralick features.

\section{Image Matching}

In this subsection, we carry out a more difficult experiment than in the previous subsections. For each image at a resolution of $4 \mathrm{~m}$, we want to find the exact same scene from the images at other resolutions with the help of wavelet features. The features are extracted and predicted as presented in Section IV-B. For an image at a resolution different from $4 \mathrm{~m}$, we search its nearest neighbor in the feature space among the set of images at $4 \mathrm{~m}$. If the two images represent the same scene, it is a correct match, otherwise it is an error. This is the same classification task as before, except that we consider each scene to be a class in itself.

In Table IV, are displayed the matching results when using features over 21 scales $\left(t_{i}=2^{i / 6}, i=0,1, \ldots, 20\right)$ and four 


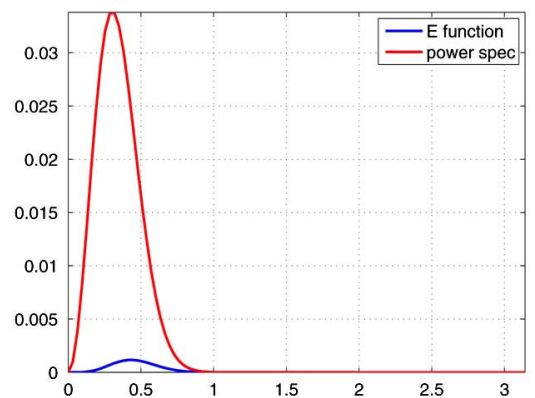

Fig. 5. Error function $(E)$ and power spectrum of wavelet coefficients $w_{r, t}$ in the frequency domain.

TABLE IV

Mismatching Percentages When Using WaVelet Features Extracted AT 21 SCALES WITH Four ORIENTATIONS

\begin{tabular}{|c|c|c|c|c|}
\hline resolution & 0.5 & 1.0 & 2.0 & 3.175 \\
\hline $\mathrm{p}=1.3$ & 4.1 & 0.27 & 0 & 1.64 \\
\hline $\mathrm{p}=0$ & 81.97 & 79.51 & 65.57 & 34.97 \\
\hline
\end{tabular}

TABLE V

Mismatching Percentages When Using WaVelet Features Extracted at THREE SCALES With Four ORIENTATIONS

\begin{tabular}{|c|c|c|c|c|}
\hline resolution & 0.5 & 1.0 & 2.0 & 3.175 \\
\hline $\mathrm{p}=1.3$ & 11.2 & 4.64 & 1.09 & 9.29 \\
\hline $\mathrm{p}=0$ & 95.90 & 96.45 & 92.62 & 62.02 \\
\hline
\end{tabular}

orientations. In Fig. 5 are displayed the matching results when using only three scales $\left(t_{i}=2^{i}, i=0,1,2\right)$.

It can be observed that:

- The errors increase considerably when compared to the classification results of the previous subsection, especially when $p=0.0$. This is due to the fact that in the image database, there are many scenes of the same class which are very similar one to another. Therefore, the points representing these images in the feature space are close one to another. As a consequence, a small error in the prediction causes an error in the image matching, in contrast with the classification case of Section V-C.

- The small errors in the case $p=1.3$ confirm the accuracy of the scheme.

\section{CONCLUSIONS AND PERSPECTIVES}

In this paper, we have proposed a scheme for comparing wavelet features from images taken at different resolutions.

The acquisition of images is modeled by a convolution followed by a sampling. The scheme to compare wavelet features is based on this model and the semi-group property of the Gaussian kernel. We first checked experimentally the validity of this scheme and showed that the numerical accuracy is significantly improved compared to a naive approach where resolution change is simply modeled by a zoom. This approach is then applied for the classification of satellite images at several resolutions simulated by the CNES. Our approach improves significantly the accuracy of the classification. This fact is confirmed trough an image matching experiment.

The method presented in Section IV-B to compare wavelet features between images with different resolution is quite gen- eral. We applied it to image classification, but other tasks could benefit from this approach. For instance, the scale invariance of wavelet transformations has been applied to the fusion of aerial images having different resolutions [30]-[32]. None of these works takes into account the influence of the optics and captors on the change of resolution. We believe that the approach presented in Section IV could improve the precision of such fusions of images.

\section{APPENDIX I}

In this Appendix, we detail the approximation made in (5) and compute the error functions of the approximation made in (6).

Recall that $w_{q, r, t}$ are the wavelet (Gaussian derivative) coefficients extracted from $f_{r}$ at scale $t$ and in direction $q$, where $f_{r}$ is the digital image at resolution $r$ obtained from the continuous scene $f$. For clarity, we only consider the 1-D case. Recall (5)

$$
\begin{aligned}
w_{q, r, t} & =\Delta_{q} \tilde{k}_{t} \tilde{*} \Pi_{r}\left(k_{r p} * f\right) \\
& =\tilde{k}_{t} \tilde{*} \Delta_{q} \Pi_{r}\left(k_{r p} * f\right) .
\end{aligned}
$$

In the $1-\mathrm{D}$ case, we have

$$
w_{r, t}=\tilde{k}_{t} \tilde{*} \Delta_{x} \Pi_{r}\left(k_{r p} * f\right) .
$$

Writing $g(x)=\left(k_{r p} * f\right)(x)$, we have

$$
\begin{aligned}
w_{r, t}(x) & =\tilde{k}_{t} \tilde{*} \Delta_{x} \Pi_{r}(g(x)) \\
& =\tilde{k}_{t} \tilde{*}\left(\Pi_{r}(g(x+r)-g(x))\right)
\end{aligned}
$$

and since $g \in C^{1}$, we have

$$
g(x+r)=g(x)+r g^{\prime}(x)+o(r) .
$$

Therefore

$$
\begin{aligned}
w_{r, t} & \approx \tilde{k}_{t} \tilde{*} \Pi_{r}\left(g(x)+r g^{\prime}(x)-g(x)\right) \\
& =\tilde{k}_{t} \tilde{*} \Pi_{r}\left(r g^{\prime}(x)\right) \\
& \approx r \tilde{k}_{t} \tilde{*} \Pi_{r}\left(k_{r p} * f^{\prime}(x)\right) .
\end{aligned}
$$

This is the approximation made in (5). Next, we compute the error energy of the approximation made in (6). For simplicity, we consider the resolution $r=1$. We want to show that the energy of the error is small

$$
\begin{aligned}
E & =\sum_{x}\left|\Delta_{x} \tilde{k}_{t} * \Pi_{1}(g)-\Pi_{1}\left(k_{t} * g^{\prime}\right)\right|^{2} \\
& =\int\left|\mathrm{FT}\left\{\Delta_{x} \tilde{k}_{t} * \Pi_{1}(g)-\Pi_{1}\left(k_{t} * g^{\prime}\right)\right\}\right|^{2} d \omega
\end{aligned}
$$

where $\mathrm{FT}(f)$ is the Fourier transform of $f$. With the approximation $k_{t} \approx \Pi_{r}\left(k_{r t}\right)$, we have

$$
\begin{aligned}
E \approx & \int\left|\mathrm{FT}\left\{\Delta_{x} \Pi_{1}\left(k_{t} * g\right)-\Pi_{1}\left(k_{t} * g^{\prime}\right)\right\}\right|^{2} d \omega \\
= & \int \mid \mathrm{FT}\left\{\Pi _ { 1 } \left(k_{t} * g(x+1)\right.\right. \\
& \left.\left.-k_{t} * g(x)\right)-\Pi_{1}\left(k_{t} * g^{\prime}\right)\right\}\left.\right|^{2} d \omega \\
= & \int \mid \mathrm{FT}\left\{\Pi _ { 1 } \left(k_{t} * g(x+1)\right.\right. \\
& \left.\left.-k_{t} * g(x)-k_{t} * g^{\prime}\right)\right\}\left.\right|^{2} d \omega .
\end{aligned}
$$


We suppose that the image is not aliased, (i.e., $k_{t} * g$ is band limited), therefore

$$
\begin{aligned}
E & \approx 2 \int_{0}^{\pi}\left|\mathrm{FT}\left\{k_{t} * g(x+1)-k_{t} * g(x)-k_{t} * g^{\prime}\right\}\right|^{2} d \omega \\
& =2 \int_{0}^{\pi}\left|e^{j \omega}-1-j \omega\right|^{2}\left|\mathrm{FT}\left\{k_{t} * g\right\}\right|^{2} d \omega \\
& =2 \int_{0}^{\pi}\left(2(1-\cos \omega-w \sin \omega)+\omega^{2}\right)\left|\mathrm{FT}\left\{k_{t} * g\right\}\right|^{2} d \omega \\
& \approx 2 \int_{0}^{\pi}\left(2\left(-\frac{\omega^{2}}{2}+\frac{\omega^{4}}{8}\right)+\omega^{2}\right)\left|\mathrm{FT}\left\{k_{t} * g\right\}\right|^{2} d \omega \\
& \approx \int_{0}^{\pi} \frac{\omega^{4}}{2}\left|\mathrm{FT}\left\{k_{t} * k_{p} * f\right\}\right|^{2} d \omega \\
& =\int_{0}^{\pi} \frac{\omega^{4}}{2}\left|\mathrm{FT}\left\{k_{\sqrt{p^{2}+t^{2}}}\right\}\right|^{2}|\mathrm{FT}\{f\}|^{2} d \omega .
\end{aligned}
$$

Recall that $t \geq 1$ and $p=1.3$ in all our numerical experiments. Since the power spectrum of the image $f$ is generally decreasing, and $\left(\omega^{4}\right) /(2)$ is increasing, the worst case (which yields the largest error) is, therefore, $|\mathrm{FT}\{f\}|^{2}=1$ for all $\omega$, where we have

$$
E \approx \int_{0}^{\pi} \frac{\omega^{4}}{2}\left|\mathrm{FT}\left\{k_{\sqrt{1^{2}+1.3^{2}}}\right\}\right|^{2} d \omega
$$

and

$$
\frac{E}{\sum\left|w_{r, t}\right|^{2}} \approx 0.035
$$

Fig. 5 shows a plot of $E$ and the power spectrum of wavelet coefficients $w_{r, t}$ in the frequency domain for the case where $|\mathrm{FT}\{f\}|^{2}=1$. In the worst case, the approximation made in (6) yields an energy error of $3.5 \%$ when compared to the energy of the original wavelet coefficients.

\section{ACKNOWLEDGMENT}

The authors would like to thank M. Datcu, A. Giros, and H. Maitre for their advice and comments.

\section{REFERENCES}

[1] D. G. Lowe, "Distinctive image features from scale-invariant keypoints," Int. J. Comput. Vis., vol. 60, no. 2, pp. 91-110, 2004.

[2] Z. Fan, F. S. Cohen, and M. A. Patel, "Classification of rotated and scaled textured images using gaussian markov random field models," IEEE Pattern Anal. Mach. Intell., vol. 13, no. 2, pp. 192-202, Feb. 1991.

[3] J. Zhang and T. Tan, "Affine invariant texture signatures," in Proc. Int. Conf. Image Processing, Thessaloniki, Greece, Oct. 2001, vol. 2, pp. 618-621.

[4] C. M. Pun and M. C. Lee, "Log-polar wavelet energy signatures for rotation and scale invariant texture classification," IEEE Pattern Anal. Mach. Intell., vol. 25, no. 5, pp. 590-603, May 2003.

[5] J. Zhang and T. Tan, "A brief review of invariant texture analysis methods," Pattern Recognit., vol. 35, pp. 735-747, 2002.

[6] B. Luo, J.-F. Aujol, Y. Gousseau, S. Ladjal, and H. Maître, "Characteristic scale in satellite images," in Proc. ICASSP, Toulouse, France, May 2006, vol. 2, pp. 809-812.

[7] B. Luo, J.-F. Aujol, Y. Gousseau, S. Ladjal, and H. Maître, "Resolution independant characteristic scale dedicated to satellite images," IEEE Trans. Image Process., vol. 16, no. 10, pp. 2503-2514, Oct. 2007.

[8] J. Flusser and T. Suk, "Degraded image analysis: An invariant approach,” IEEE Pattern Anal. Mach. Intell, vol. 20, no. 6, pp. 590-603, Jun. 1998.

[9] J. van de Weijer and C. Schmid, "Blur robust and color constant image description," presented at the Int. Conf. Image Processing, 2006.
[10] A. Lorette, X. Descombes, and J. Zerubia, "Texture analysis through a 'du markovian modelling and fuzzy classification: Application to urban area extraction from satellite images," Int. J. Comput. Vis., vol. 36, no. 3, pp. 221-236, 2000.

[11] G. Rellier, X. Descombes, F. Falzon, and J. Zerubia, "Texture feature analysis using a Gauss-Markov model in hyperspectral image classification," IEEE Trans. Geosci. Remote Sens., vol. 42, no. 7, pp. 1543-1551, Jul. 2004.

[12] M. Datcu, K. Seidel, and M. Walessa, "Spatial information retrieval from remote-sensing images, II. Information theoretical perspective," IEEE Trans. Geosci. Remote Sens., vol. 36, no. 5, pp. 1431-1445, May 1998.

[13] K. Chen and R. Blong, "Identifying the characteristic scale of scene variation in fine spatial resolution imagery with wavelet transformbased sub-image statistics," Int. J. Remote Sens., vol. 24, no. 9, pp. 1983-1989, 2003.

[14] T. Gevers and H. Stokman, "Robust photometric invariant region detection in multispectral images," Int. J. Comput. Vis., vol. 53, pp. 135-151, Jul. 2003.

[15] D. Van Dyck, G. Van de Wouwer, and G. Scheunders, "Statistical texture characterization from discrete wavelet representations," IEEE Trans. Image Process., vol. 8, no. 4, pp. 592-598, Apr. 1999.

[16] M. N. Do and M. Vetterli, "Wavelet-based texture retrieval using generalized gaussian density and Kullback-Leibler distance," IEEE Trans. Image Process., vol. 11, no. 2, pp. 146-158, Feb. 2002.

[17] J.-F. Aujol, G. Aubert, and L. Blanc-Féraud, "Wavelet-based level set evolution for classification of textured images," IEEE Trans. Image Process., vol. 12, no. 12, pp. 1634-1641, Dec. 2003.

[18] M. Unser, "Texture classification and segmentation using wavelet frames," IEEE Trans. Image Process., vol. 4, no. 11, pp. 1549-1560, Nov. 1995

[19] H. Choi and R. G. Baraniuk, "Multiscale image segmentation using wavelet-domain hidden Markov models," IEEE Trans. Image Process., vol. 10, no. 9, pp. 1309-1321, Sep. 2001.

[20] G. Van de Wouwer, P. Scheunders, and D. Van Dyck, "Statistical texture characterisation from discrete wavelet representation," IEEE Trans. Image Process., vol. 8, no. 4, pp. 592-598, Apr. 1999.

[21] J. Liu and P. Moulin, "Information-theoretic analysis of interscale and intrascale dependencies between image wavelet coefficients," IEEE Trans. Image Process., vol. 10, no. 11, pp. 1647-1658, Nov. 2001.

[22] J. L. Chen and A. Kundu, "Rotation and gray scale transform invariant texture identification using wavelet decomposition and hidden Markov model," IEEE Trans. Pattern Anal. Mach. Intell., vol. 16, no. 2, pp. 208-214, Feb. 1994.

[23] S. W. Myint Nina, S.-N. Lam, and J. M. Tyler, "Wavelets for urban spatial feature discrimination: Comparisons with fractal, spatial autocorrelation, and spatial co-occurence approaches," Photogramm. Eng. Remote Sens., vol. 70, pp. 803-812, July 2004.

[24] B. Luo, J.-F. Aujol, Y. Gousseau, and S. Ladjal, "Interpolation of wavelet features for satellite images with different resolutions," presented at the IGARSS, Denver, CO, 2006.

[25] D. F. Ellliott, L. L. Hung, E. Leong, and D. L. Webb, "Accuracy of gaussian approximation for simulating EO sensor response," in Proc. Conf. Record 30th Asilomar Conf. Signals, Systems and Computers, 1996, pp. 868-872.

[26] B. Zhang, J. Zerubia, and J. C. Olivo-Marin, "Gaussian approximations of fluorescence microscope point-spread function models," Appl. Opt., vol. 46, no. 10, pp. 1819-1829, Apr. 2007.

[27] S. G. Mallat, "A theory for multiresolution signal decomposition: The wavelet representation," IEEE Pattern Anal. Mach. Intell., vol. 11, no. 7, pp. 674-693, Jul. 1989.

[28] S. G. Mallat, A Wavelet Tour of Signal Processing. New York: Academic, 1998.

[29] R. M. Haralick, K. Shanmugam, and I. Dinstein, "Textural features for image classification," IEEE Trans. Syst., Man, Cybern., vol. SMC-3, no. 6, pp. 610-621, Nov. 1973.

[30] T. Ranchin, "Remote sensing and image fusion methods: A comparison," presented at the IEEE Int. Conf. Acoustics, Speech and Signal Processing, Toulouse, France, May 2006.

[31] G. Hong and Y. Zhang, "High resolution image fusion based on wavelet and ihs transformations," in Proc. 2nd GRSS/ISPRS Joint Workshop on Remote Sensing and Data Fusion Over Urban Areas, May 2003, pp. 99-104.

[32] J. Núnez, X. Otazu, O. Fors, A. Prades, V. Pala, and R. Arbiol, "Multiresolution-based image fusion with additive wavelet decomposition," IEEE Trans. Geosci. Remote Sens., vol. 37, no. 3, pp. 1204-1211, May 1999, part I. 


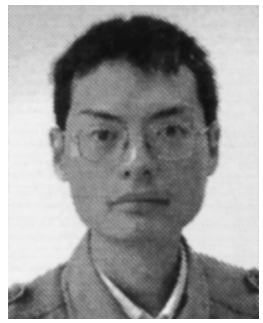

Bin Luo received the B.S. degree in telecommunications from Wuhan University, Wuhan, China, in 2003, the M.S. degree in applied mathematics and computer vision from the École Normale Supérieure de Cachan, Cachan, France, in 2004, and the Ph.D. degree in signal and image processing from the École Nationale Supérieure des Télécommunications, Paris, France, in 2007.

$\mathrm{He}$ is now a Postdoctorate at GIPSA-Lab, Grenoble, France. His research interests include remote sensing image formation, data mining, image processing, and computer vision.

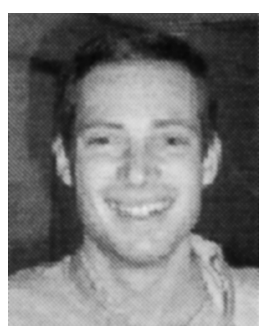

Jean-François Aujol studied mathematics at the Ecole Normale Supérieure, Cachan, France, and received the Ph.D. degree in applied mathematics from Nice-Sophia-Antipolis University, France, in June 2004.

In 2004-2005, he was successively an Assistant Researcher in the Applied Mathematics Department, University of California, Los Angeles, and then a Research Engeneer at ENST (TSI Department), Paris, France. Since then, he has been a Scientist Researcher with CNRS at the Centre de Mathématiques et de

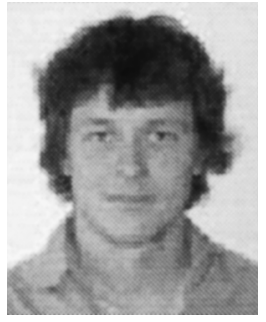
puter vision, and image processing.
Yann Gousseau graduated from the École Centrale de Paris, France, in 1995, and received the Ph.D. degree in applied mathematics from the University Paris Dauphine in 2000.

After working as a Postdoctorate at Brown University, Providence, RI, and at the École Normale Supérieure de Cachan, France, he joined the École Nationale Supérieure des Telécommunications, Paris, France, as an Associate Professor in 2001.

His research interests include the mathematical modeling of natural images, image analysis, com-

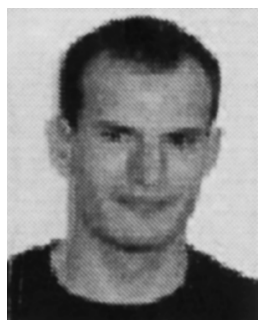

Saïd Ladjal is a former student of the École Normale Supérieure, France. He received the engineering degree from the ENST, France, and the Ph.D. degree from the ENS Cachan, France, in 2005.

He is currently an Associate Professor in the Signal and Image Processing (TSI) Department, ENST. His research intersets include image restoration and blind deconvolution.

leurs Applications (CMLA, ENS Cachan, CNRS, PRES UniverSud), France.

$\mathrm{He}$ is interested in mathematical problems related to image processing. 